\title{
Una breve mirada hacia los antecedentes teóricos de la Pedagogía Social
}

\author{
Ruth Molina Vasquez*
}

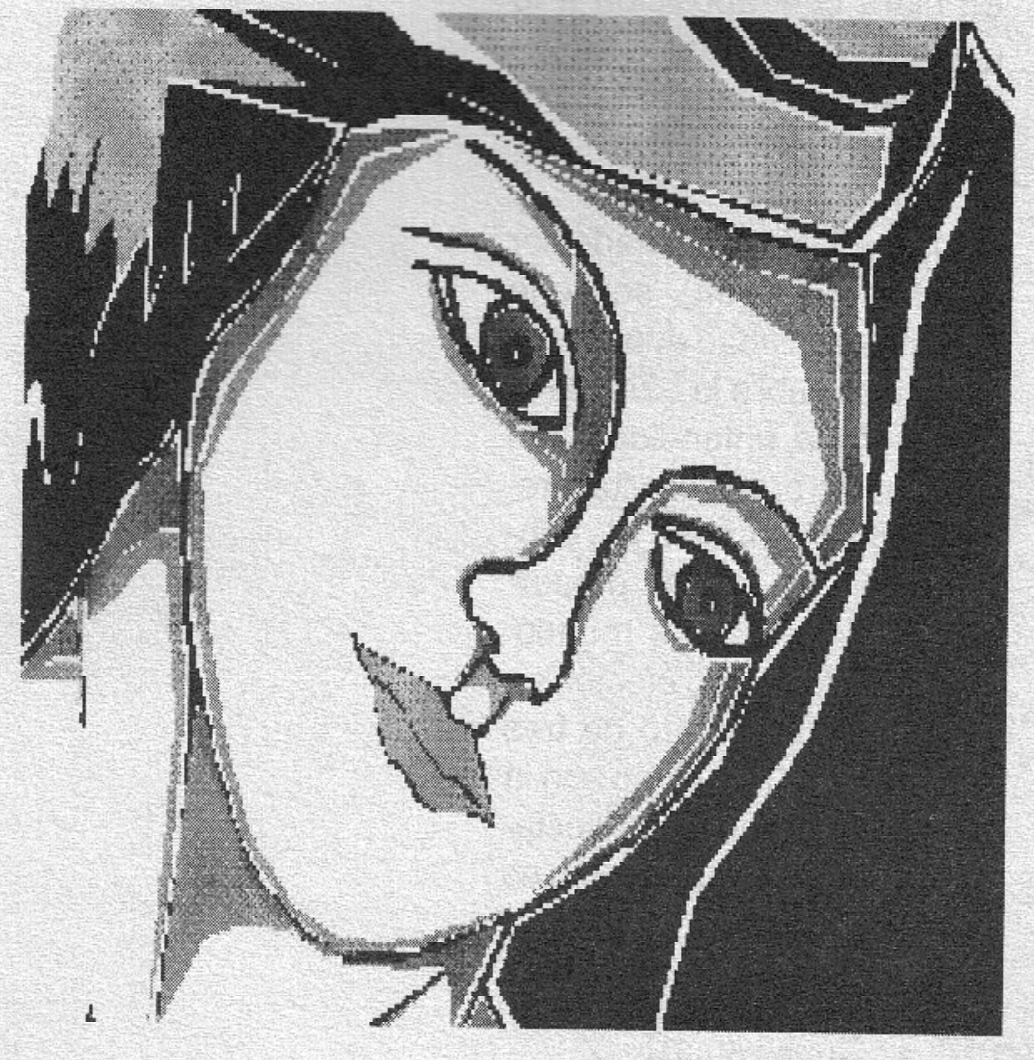

\section{Introducción}

En torno a la reflexión sobre el modelo pedagógico que la Facultad de Educación de la Corporación Universitaria Minuto de Dios adopte en la construcción de su proyecto educativo de formación de docentes, cobra importancia la mirada histórica sobre la conformación del concepto de Pedagogía Social, sus connotaciones y las características del manejo que se le da en la actualidad. Este escrito pretende abrir una discusión al interior de la Facultad sobre la adopción o adaptación de una pedagogía - que bien podría ser la Pedagogía Social -, que caracterice 10 que hasta el momento ha sido la práctica pedagógica no sólo al interior de la Universidad, sino en toda la experiencia de la Organización Minuto de Dios.

En esta discusión están llamados a participar los docentes, los estudiantes, los egresados, el cuerpo administrativo, la comunidad de EI Minuto de Dios y todos aquellos que de una u otra manera participan en la construcción de nuestro Proyecto Pedagógico.

\footnotetext{
- Ruth Molina Vasquez es Licenciada en Psicología y Pedagogía de la UPN. Magister en Informática
} aplicada a la educación. Actual Coordinadora del área de Estética en la Facultad de Educación. 


\section{Historia de la Pedagogía Social}

El concepto de pedagogia social tuvo su origen en la Alemania del siglo XIX, período durante el cual fue considerada como una ciencia de ayuda a la juventud,entendida en tres sentidos diferentes: ayuda educativa, profesional y cultural. Aunque sus precedentes son muy remotos, pueden situarse en Pestalozzi y Froebel, si bien de igual manera los cambios bruscos provocados por la industrialización y la situación socio - política, determinaron la aparición de una manera nueva de dar solución a las necesidades sociales, cambiando el concepto de caridad por el de justicia, lo cual quedó inmerso en la creación del Partido Social Demócrata Alemán en 1878. Entre las tensiones del poder reinante y la social democracia, la Pedagogía Social se mostró como una opción burguesa con la cual ésta se defendía del proletarismo naciente aunque buscara mejorar el nivel educativo de los trabajadores.
La Pedagogía Social que nace con Karl Friedrich Magers y cuyo concepto es dado por Adolf Diesterweg entre 1849 y 1850 , tiene su representante más importante en P. Natorp, quien en 1898 publica el libro "Pedagogía social", en el que expone los conceptos que la componen. En clara contraposición a lo que hasta el momento se venía gestando en el campo pedagógico del individualismo, herencia de Locke y Rousseau, Natorp (citado por FERMOSO, 1994), sostiene que toda actividad educadora se realiza sobre la base de la comunidad, entendida como una unidad orgánica vital presidida por la armonía y la concordancia, la cual le proporciona una visión de mundo. Toda educación se efectúa en la comunidad dentro de tres grandes círculos como son la familia, la escuela y la sociedad, por tanto la formación es un bien común a todos los individuos. Natorp, fue el primero en intentar elaborar una teoría de la Pedagogía Social que apoyara su carácter práctico, por lo que formuló objetivos como la creación de una conciencia comunitaria contra el egoismo y la conciencia de clase, la superación de la desunión del pueblo alemán, la eliminación del vacío entre clases sociales y la democratización de la educación.

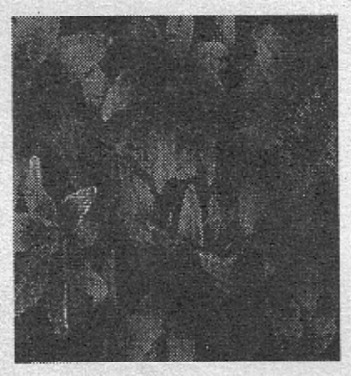


A comienzos del siglo $X X$, el término es utilizado originalmente, para referirse a todos los movimientos alemanes que se dedicaban a la ayuda de pobres, desvalidos y abandonados, debido a que en su segunda etapa, H. Nohl, pedagogo de la Universidad de Gotinga, la caracterizara como la pedagogía del "tercer espacio", ya que no se realiza ni en la familia ni en la escuela y sus destinatarios fueron obreros y jóvenes. En ese momento histórico, la pedagogía social se basa en la construcción del individuo, la cual no se puede separar de la construcción y reconstrucción social condicionada por las situaciones comunitarias en las que se desenvuelve el hombre; por lo tanto, sus objetivos son la prevención de problemas sociales, la intervención y la curación de necesidades.

El Nacionalsocialismo que dominó a Alemania durante la segunda guerra mundial, marcó el decaimiento de la Pedagogía Social, gracias a la falta de apoyo estatal, el cual reaparece en la postguerra mendiante la ayuda de organismos internacionales, la creación de nuevas instituciones pedagógicas de albergue y cuidado de niños y jóvenes y el desarrollo de una legislación social hacia la democratización.
En otros paises como Italia, la Pedagogía Social se entiende como una educación social informal y extra - escolar, atendida principalmente por los medios de comunicación, mientras que en los países anglosajones se busca a través de ella el análisis y la acción en contra de la pobreza, la criminalidad juvenil y el alcoholismo, lo cual conlleva a que dificilmente se establezcan límites entre esta y el trabajo social. En Francia se han organizado programas de intervención en la solución a necesidades sociales en los frentes de animación socio cultural, educación de adultos, adaptación y formación en la empresa, los cuales han tenidos influencia también en España, en donde se han adoptado programas de formación al rededor de esta temática, aunque la historia de la pedagogía social como tal en este país, empieza en el año de 1944, cuando es incluida en el plan de estudios de la sección de Pedagogía de la Universidad de Madrid y en años posteriores en las Universidades de Barcelona, Valencia, Santiago de Compostela y Murcia, a nivel de seminarios de pregrado, diplomado y especialidad. Es en esta última en donde se empieza a editar la Revista de Pedagogia Social en el año de 1986. 
La evolución histórica de lo que se concibe como Pedagogía Social, se ha derivado en cuatro tendencias teóricas, algunas de ellas vigentes en la actualidad: teoría crítica, racionalismo crítico, marxismo y ecologismo.

Dentro de la tendencia de la teoría crítica se dan cinco corrientes diversas entre las cuales la Pedagogía Social es entendida como:

\section{a) Ayuda a la juventud:} Plantea una censura a la sociedad pues es quien genera las grandes necesidades de la juventud y propicia los fenómenos de inadaptación, disocialización y otros traumas.

\section{b) Trabajo juvenil anticapi-} talista:Este movimiento, nacido dentro de la izquierda crítica, contempla aspectos como el análisis de formas políticas y perspectivas agresivas en diversos espacios y de experiencias y acciones, polémicas, prácticas y dificultades de pedagogos políticamente beligerantes, la extracción de conclusiones y objetivos útiles al trabajo de los pedagogos socialistas y la elaboración de informes sobre las luchas de la juventud. c) Higiene social: Su representante G. Iben, toma la Pedagogía Social como una ciencia autónoma cuyos objetos son la investigación, la formación práctica y la construcción teórica de ayuda a procesos educativos insatisfactorios en situaciones sociales de indigencia.

\section{d) Trabajo social crítico del} círculo de trabajo: Servicio de información ya desaparecido, acerca de la práctica sociopedagógica.

e) Emancipación: Este movimiento derivado de la teoría crítica de la escuela de Frankfurt, busca la emancipación como interés fundamental dentro de la ciencia de la educación. Su principal representante, Klaus Mollenhauer, plantea como tesis principales un interés epistemológico referido al cambio y las formas de praxis a través del cambio de condicionamientos sociales, la eliminación del dominio racional y toda forma de coacción, la educación fuera de la escuela y con la participación de los educandos, la confrontación de lo fáctico con lo posible objetivo y intelección de la crítica en el sentido práctico y no ontológico. 
La tendencia del Racionalismo Crítico, como modelo sociopedagógico antimarxista y opuesto a la escuela de Franciort, se fundamenta en el neopositivismo y sus tesis principales sostienen que la ciencia no es normativa, por tanto debe estar libre de juicios de valor y debe convertirse en un saber tecnológico, el objeto de todo conocimiento científico debe ser empíricamente falsable y las hipótesis comprobables se pueden deducir a partir de proposiciones básicas. Su principal representante Lutz Rössner, comenzó a elaborar en 1966 una nueva teoría sobre la ciencia del trabajo social, a partir de un modelo en el que puede tener sentido la Pedagogia Social que en cuanto a saber científico, se caracteriza por ser un saber metafísico que se debe elaborar al modo de las ciencias naturales, y debe ser probado empíricamente. Su objeto de estudio es la praxis educativa y las necesidades sociales de las cuales investiga las relaciones causales.

En la tendencia del materialismo histórico o marxismo, la Pedagogía Social utiliza como base metodológica el materialismo dialéctico para fusionar la teoría y la práctica con el objetivo de interpretar las necesidades e intereses de la clase trabajadora, ya que se concibía la teoría como un medio de conocimiento $y$ cambio. Esta concepción de Pedagogía Social busca establecer una lucha contra la miseria social la cual es integrada al movimiento de clases, conducir una conciencia desde esa miseria social y la desclasación de la población de trabajadores hacia su organización, contribuir al cambio de la clase trabajadora en una clase para si, cambiar la conciencia subjetiva de los propios intereses hacia una conciencia de clase que reconozca los intereses comunes como intereses del colectivo que se traduce en una lucha de clases. La educación por tanto, es concebida como una lucha de clases a la luz de la política y la agitación revolucionaria, en donde la Pedagogía Social ha de producir una concientización y una activación adecuada de los aprendizajes de los trabajadores, quienes han de luchar en contra de la pobreza y el sistema capitalista de producción.

La tendencia ecologista de la pedagogia social tiene sus raices en la tendencia fenomenológica de $E$. Husserl, aplicada en la segunda mitad del presente siglo y que toma diferentes matices como los de "modelo ecológico" o "modelo del mun- do vital". Esta tendencia busca desarrollar investigaciones en diferentes líneas, como las interacciones entre el individuo y su medio, con preferencia especial por los problemas de la vida que se originan en las relaciones interpersonales, los espacios vitales reducidos, los problemas y necesidades relacionados con el fin vital de las personas en momentos de transición vital, (como cambios de status, rol, dificultades de grupo, etc.), los problemas relacionados con el medio físico y social (como vivienda, instituciones sociales, etc.) y, necesidades y problemas generados por las interacciones humanas y las comunicaciones entre las personas y los grupos.

\section{Concepto de Pedagogía Social}

A través de la historia han sido muchas y variadas las concepciones que se han tenido de lo que es la Pedagogía Social. Entre estas adquieren importancia las de Mollenahuer (Citado por FERMOSO, 1994), quien clasifica la Pedagogía Social de acuerdo a cuatro concepciones diferentes: como ciencia de los fines diferenciados de la educación y de sus ideales, 


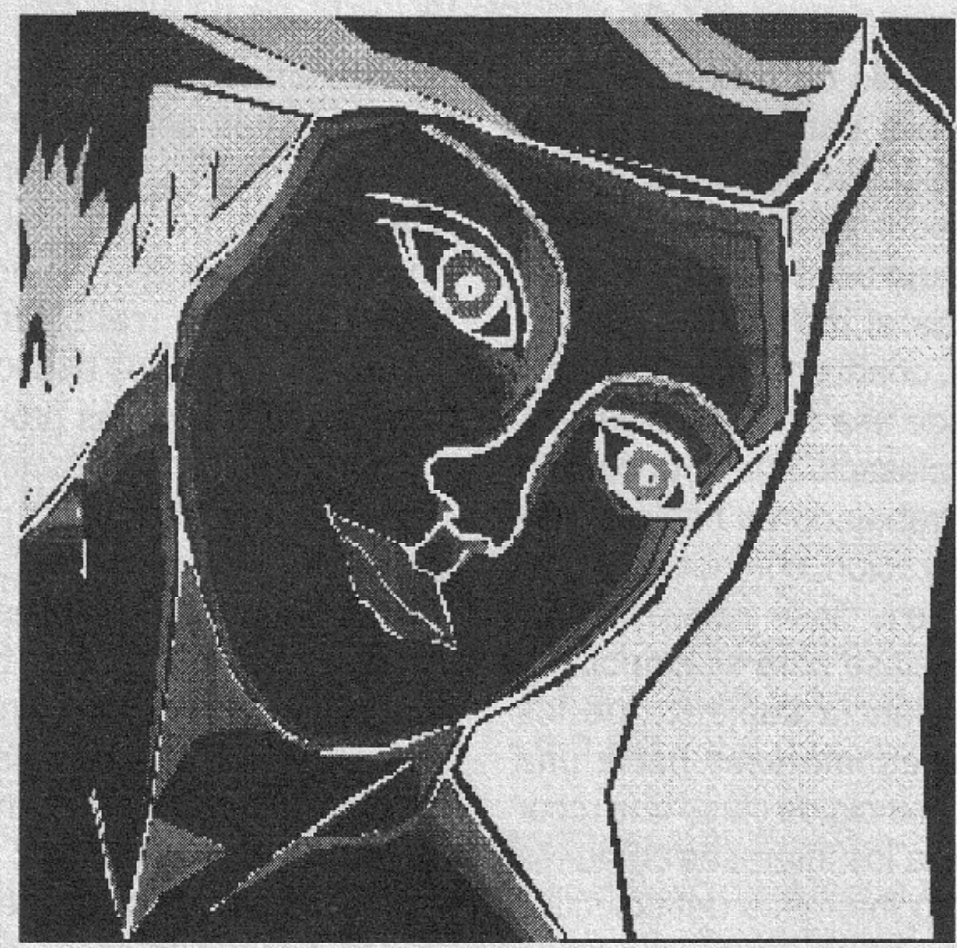

como aplicación de una ética social determinada, como acentuación de los objetivos generales de la pedagogía y como un aspecto educativo especial que se estudia mediante la estructura de grupos.

J.M. Quintana, citado por el mismo autor, reitera la importancia de hacer una clasificación conceptual del término y aporta cinco concepciones diferentes, aunque admite que las más acertadas en su criterio son: la Pedagogía Social como doctrina de formación social del individuo y como doctrina del sociologismo pedagógico. Esta última concepción pone de relieve nue- vamente la dualidad del papel de la sociedad y la educación: la primera con funciones de carácter educativo y la segunda como una entidad socializadora.

Para efectos de la presente reflexión, se propone adoptar el concepto de Pedagogía Social como una ciencia práctica, social y educativa que busca fundamentar, justificar y comprender la normatividad o los principios más adecuados para realizar una labor de intervención, ayuda y reinserción social de quienes padecen deficiencias de socialización o insatisfacción de las necesidades bá- sicas amparadas por los derechos humanos. (FERMOSO, 1994).

En este orden de ideas, la Pedagogía Social es concebida como una ciencia práctica, en tanto posee un cuerpo teórico de conocimientos de rango epistemológico científi$\mathrm{co}$, que posibilita su aplicabilidad, como una ciencia social no formal que busca fundamentar y justificar el porqué del conocimiento científico en educación dentro de la concepción de racionalismo crítico y comprender, más no establecer la normatividad más adecuada para llevar a cabo una práctica en el ámbito de acción de la intervención social. Se le puede llamar también "pedagogía de la necesidad", puesto que busca identificar y atender a quienes sufren de carencias, desajustes, marginaciones o inadaptaciones sociales contempladas en los derechos humanos, en los sectores tanto de la infancia como la juventud, la edad adulta y la tercera edad. 

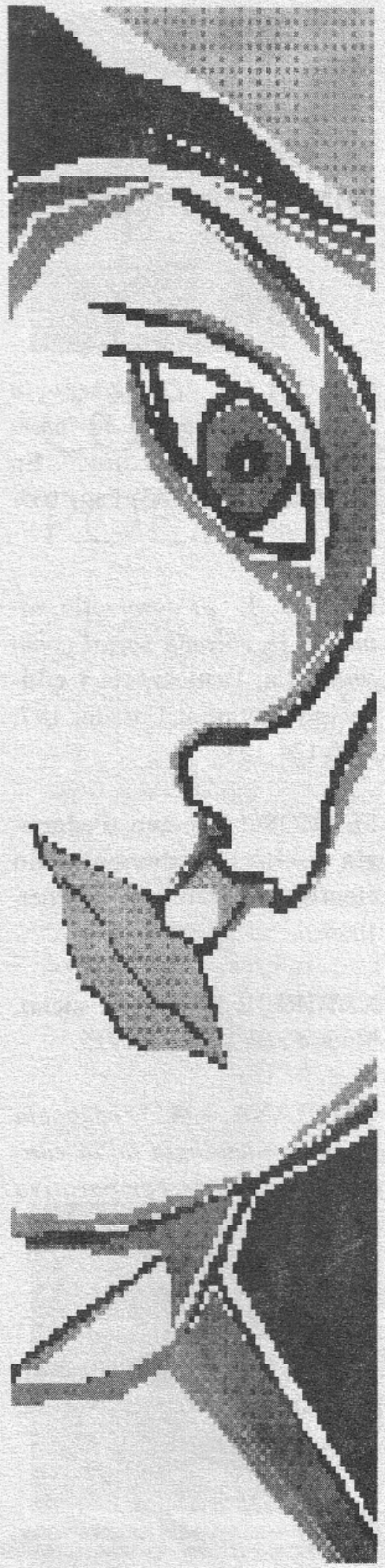

3. Pedagogía Social en la Corporación Universitaria Minuto de Dios

Dentro del contexto teórico y práctico de la Corporación Universitaria Minuto de Dios, la Pedagogía Social posee unas características particulares, en tanto se le considera como una ciencia educativa y práctica que implica la intervención social en individuos y comunidades preferenciaimente de pobres y jóvenes, quienes padecen necesidades básicas, carencias o marginaciones.

En este contexto, el objetivo de la Pedagogía Social es la fundamentación, justificación, comprensión e implementación de soluciones a los problemas sociales de las comunidades intervenidas, a través de la práctica educativa en la autoformación y autogestión, desde una doble mirada: la interpretación de las necesidades y la intervención social.

Esta intervención social se realiza desde tres aspectos diferentes y complementarios: la Formación Integral de estas comunidades en el amor, la doctrina social de la Iglesia y la espiritualidad eudista; en el desarrollo de competencias personales y profesionales, de capacidades prácticas, destrezas técnicas, de una visión ética de responsabilidad social y el cultivo de la sensibilidad; la Investigación como un proceso interpretación de la realidad, de constante reflexión sobre la producción de conocimiento y su aplicabilidad en el campo social de acuerdo con las necesidades de las comunidades intervenidas; $y$ finalmente, la Proyección Social enfatizada en el desarrollo humano y el aporte de nuevas prácticas sociales en la solución a necesidades comunitarias y el reconocimiento y respeto de sus valores propios, para contribuir en la construcción de una nueva sociedad.

Desde el punto de vista de la Formación Integral, las funciones de la Pedagogía Social se encaminan a brindar oportunidades de formación profesional y personal, preferencialmente a jóvenes de sectores marginados, como innovadores sociales, es decir, sujetos capaces de gestar procesos de desarrollo comunitario por medio de la autogestión, de participar activamente en procesos democráticos y de solidarizarse ante las condiciones adversas de vida de las personas y comunidades con las que convive y trabaja. 
En la investigación, la Pedagogía Social tiene entre sus funciones la de determinar las características de su acción educativa, fundamentar teóricamente los procesos de intervención, sistematizar y validar la práctica social, detectar problemas, plantear hipótesis de solución y confrontarlos de forma participativa con la realidad.

Las funciones de la Pedagogía Social desde el aspecto de intervención social son las de proporcionar acciones de anticipación, ayuda y solución de necesidades comunitarias por medio de la fundamentación, justificación y comprensión de problemas generados en la dinámica social.

\section{Se abre la dis- cusión}

La perspectiva planteada hasta aquí, muestra como la fundamentación histórica y teórica de lo que a nivel mundial se entiende por Pedagogía Social, tiene una amplia influencia en la práctica social que ha venido desarrollando la Organización Minuto de Dios y particularmente la $\mathrm{Fa}$ cultad de Educación de la Corporación Universitaria Minuto de Dios. Sin embargo por otro lado, es necesario

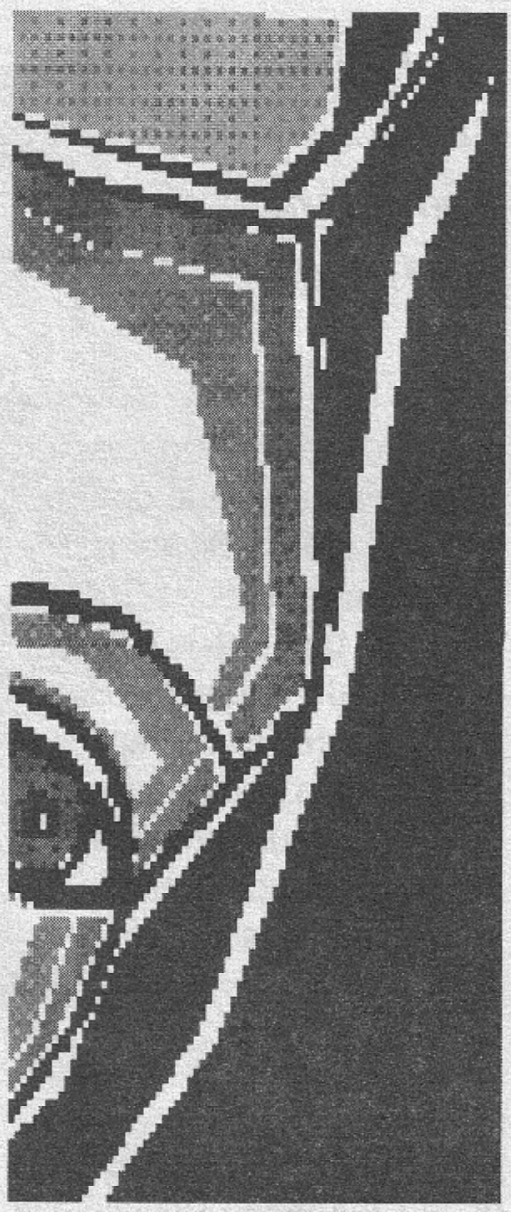

contemplar las particularidades de esta práctica y reflexionar sobre las especiales características que posee. Ello invita a abrir una reflexión profunda y una línea de trabajo que aborde elementos acerca de la forma como la Pedagogía Social puede pasar de ser un modelo asumido en los campos del Trabajo Social, para convertirse en un Modelo Pedagógico -con todo lo que ello implica-.Esta construcción debe caracterizarse por ser un trabajo de construcción colectiva en la que todos están invitados a participar.

\section{Referençias bibliográficas}

COLOM,A.J. "La pedagogía social como modelo de intervención socio-educativa". En Bordón No 35(1983) Pag. 165180.

DEWEY, J. "El desarrollo natural y la eficacia social como fines". En: Democracia y educación. Editorial Losada, 197, Pag. 124-137.

FERMOSO, Paciano. Pedagogía Social: Fundamentación científica. Barcelona: Herder, 1994.

NATORP, P. Pedagogía social. Madrid : La Lectura, 1915.

QUINTANA, J.M. "Pedagogía social y sociología de la educación: análisis comparativo de sus conceptos". En: Perspectivas pedagógicas. $\mathrm{N}^{\circ} 39$ 40 (1977). Pag. 303 - 313.

La investigación participativa. Madrid : Narcea, 1986. 